\title{
Ground State of Beryllium Atom Using Variational Monte Carlo Method
}

\author{
S.B. DOMA ${ }^{a, *}$, H.S. EL-GENDY ${ }^{b}$, \\ M.A. ABDel-KhaleK ${ }^{a}$ And M.E. Mohamed ${ }^{a}$ \\ ${ }^{a}$ Faculty of Science, Alexandria University, Egypt \\ ${ }^{b}$ College of Science and Humanities, Shaqra University, KSA
}

Received: 19.06.2020 \& Accepted: 02.09.2020

Doi: 10.12693/APhysPolA.138.838 *e-mail: sbdoma@alexu.edu.eg

\begin{abstract}
The variational Monte Carlo method is applied to calculate the ground-state energy eigenvalues and eigenfunctions of a beryllium atom. The obtained wave functions are used to calculate the mean distance of the electrons from the nucleus and the mean distance between the electrons. The computations were carried out with the trial wave functions in the form of the Slater determinant multiplied by a correlation function corresponding to the interactions between the electrons. Two different kinds of orbital functions as well as two different types of correlation functions are used in the calculations. The obtained results of the ground-state energy eigenvalues and radii are very satisfactory when compared with the exact values and the previous findings.
\end{abstract}

topics: beryllium atom, variational Monte Carlo method, trial wave functions, correlation functions

\section{Introduction}

There are many kinds of approximate methods which involve minutely the Schrödinger equation of the many-electron systems. Among them are the variational method [1], the perturbation method [2], the Hartree-Fock (HF) method [3], the ab initio HF (RHF) method [4], the configuration interaction (CI) method [5], which is a post-HF linear variational method, the diffusion Monte Carlo method [6] and the variational Monte Carlo (VMC) method [7]. In the case of the VMC method, its proficiency has been proven in many calculations of atoms and molecules. The VMC method is based on incorporating the variational principle and the estimation of high-dimensional integrals by sampling the integrands using a set of randomly generated points. As a result, the integrals converge faster using the VMC technique than more conventional techniques based on sampling the integrands on a regular grid for problems involving more than a few dimensions. Moreover, the statistical error in the estimate of the integral decreases as the square root of the number of points sampled, irrespective of the dimensionality of the problem. Thus, many studies take advantage of using the VMC method to study the atomic and molecular properties. Let us shed light on the most important ones.

Sa nu-Ginarte et al. [8] calculated the ground state of $\mathrm{Be}$ and its first excited states by using the direct variational method and considering the systematic asymmetric nature of the trial wave function. A cutoff function was added to ensure confinement boundary conditions and a trial wave function was built up from hydrogenic functions. However, the interaction between the electrons was ignored in [8], thus the correlation functions were not used at all. On the other hand, $\mathrm{Wu}$ and Meng [9] applied the perturbation theory to the ground-state energy of a beryllium atom by incorporating double parameters in the Hamiltonian. The eigenvalues were determined then with a double-fold perturbation scheme, where the spin-spin interaction of electrons from different shells of the atom was also considered. The obtained ground-state energy result was in satisfactory agreement with the experiment. Also, it was found that the Coulomb repulsion of the inner-shell electrons enhances the effective nuclear charge seen by the outer-shell electrons and the shielding effect of the outer-shell electrons to the nucleus was also notable compared with that of the inner-shell electrons.

Doma et al. [10] applied the VMC method to investigate the total energies of the excited states of the helium atom. The strong magnetic field effect on the energy was investigated there and illustrated graphically. The results were in good agreement with the most recent accurate and exact values. Umrigar et al. [11] proposed modifications to the simple diffusion Monte Carlo algorithm that greatly reduces the time-step error. The improved algorithm had the time-step error smaller by a factor of 70 to 300 in the energy of $\mathrm{Be}, \mathrm{Li}_{2}$ and Ne. For other observables, the improvement was even larger. The possible effective time step with the improved algorithm was typically a factor of a few hundred 
larger than the time step used in the Green's function Monte Carlo domain. In addition, they presented optimized 109 parameters of the trial wave function for Be used in combination with their algorithm and an exceedingly accurate ground-state energy was obtained. In [12], for example, the VMC was applied to compute the energies of the $1^{1} 0^{+}$ state, the derivatives of the total energy and the ionization energies of $\mathrm{He}$ and a hydrogen negative ion in the magnetic field regime $0-10$ a.u. The calculations were based on two types of compact and accurate trial wave functions which at first were used in the calculation of energies in the absence of the magnetic field. The results were in good agreement with the most recent results. Furthermore, Alexander and Coldwell [13] used the VMC method with the features of 118 trial wave function forms for selected ground and excited states of helium, lithium and beryllium atoms. The purpose was to determine which characteristics give the most rapid convergence toward the exact non-relativistic energy. It turned out that fully antisymmetric functions are more accurate than those which use determinants and that exponential functions are more accurate than linear ones.

Further, the VMC method was also applied to investigate the ground state and some excited states of the lithium atom and its ions [14] up to $Z=10$ in the presence of an external magnetic field regime with $\gamma \simeq 0-100 \mathrm{a} . \mathrm{u}$. The effect of increasing the field strength on the ground-state energy was investigated and precise values for the crossover field strength were reported. The calculations were based on using accurate forms of trial wave functions which were put forward in calculating energies in the absence of the magnetic field. Furthermore, the value of $\gamma$ at which the groundstate energy of the lithium atom approaches zero was calculated. The obtained results were in good agreement with the most recent and exact values. Another application of the VMC method followed to light molecules [15] to evaluate the ground-state energy of a confined hydrogen molecule $\mathrm{H}_{2}$. Moreover, in [16], the binding energies of the hydrogen molecule and its molecular ion in the presence of an aligned magnetic field regime between 0 and 10 a.u. were computed with the use of the VCM. The calculations were based on two types of compact and accurate trial wave functions which were used in calculating energies in the absence of the magnetic field. Finally, Frolov and Wardlaw [17] developed a variational method which facilitates the construction of very compact and relatively accurate wave functions for four-electron atomic systems. In contrast with the remaining methods, the procedure is relatively simple in application and can be used for an arbitrary four-electron atomic system. Again, the results were in good agreement with the most recent and exact values.

In the present paper, we applied the VMC method to compute the ground-state energy of the beryllium atom. For this, we constructed the wave function using the Slater determinant (SLD) with two different correlation functions. In addition, we used modified orbital hydrogenic wave functions in the SLD with one of the used correlation functions. With the use of the constructed wave functions, the mean distance of the electrons from the nucleus and the mean distance between the electrons were calculated.

\section{Hamiltonian of the system}

The non-relativistic Hamiltonian in the infinite nuclear mass approximation for four electrons in the field of a nucleus of charge $Z$ (in the Hartree atomic units) is given by

$$
H=-\frac{1}{2} \sum_{i=1}^{4} \nabla_{i}^{2}-\sum_{i=1}^{4} \frac{Z}{r_{i}}+\sum_{i<j}^{4} \frac{1}{r_{i j}},
$$

where $\nabla_{i}$ is the vector momentum of the $i$-th electron, $Z$ is the nuclear charge $(Z=4), r_{i}$ is the distance between the $i$-th electron and the nucleus of Be and $r_{i j}$ is the distance between the $i$-th and the $j$-th electron. It is convenient to transform the kinetic energy part of (1) into the mutually independent distance coordinates, $r_{i}, r_{i j}$ and the functions of the polar angles of the electrons of the nucleus, $\theta_{i}$ and $\phi_{i}$. The general Hamiltonian in the Hylleraas coordinates is then [18]:

$$
\begin{aligned}
H & =-\frac{1}{2} \sum_{i=1}^{4} \frac{\partial^{2}}{\partial r_{i}^{2}}-\sum_{i=1}^{4} \frac{1}{r_{i}} \frac{\partial}{\partial r_{i}}-\sum_{i=1}^{4} \frac{Z}{r_{i}}+\sum_{i<j}^{4} \frac{1}{r_{i j}}-\sum_{i<j}^{4} \frac{\partial^{2}}{\partial r_{i j}^{2}}-\sum_{i<j}^{4} \frac{2}{r_{i j}} \frac{\partial}{\partial r_{i j}} \\
& -\frac{1}{2} \sum_{i \neq j}^{4} \frac{r_{i}^{2}+r_{i j}^{2}-r_{j}^{2}}{r_{i} r_{i j}} \frac{\partial^{2}}{\partial r_{i} \partial r_{i j}}-\frac{1}{2} \sum_{i \neq j}^{4} \sum_{k>j}^{4} \frac{r_{i j}^{2}+r_{i k}^{2}-r_{j k}^{2}}{r_{i j} r_{i k}} \frac{\partial^{2}}{\partial r_{i j} \partial r_{i k}}-\frac{1}{2} \sum_{i=1}^{4} \frac{1}{r_{i}^{2}} \frac{\partial^{2}}{\partial \theta_{i}^{2}} \\
& -\frac{1}{2} \sum_{i=1}^{4} \frac{1}{r_{i}^{2} \sin ^{2}\left(\theta_{i}\right)} \frac{\partial^{2}}{\partial \varphi_{i}^{2}}-\sum_{i \neq j}^{4}\left(\frac{r_{j}}{r_{i} r_{i j}} \frac{\cos \left(\theta_{j}\right)}{\sin \left(\theta_{i}\right)}+\frac{\cot \left(\theta_{i}\right)}{2} \frac{r_{i j}^{2}-r_{i}^{2}-r_{j}^{2}}{r_{i}^{2} r_{i j}}\right) \frac{\partial^{2}}{\partial \theta_{i} \partial r_{i j}} \\
& -\frac{1}{2} \sum_{i=1}^{4} \frac{\cot \left(\theta_{i}\right)}{r_{i}^{2}} \frac{\partial}{\partial \theta_{i}}-\sum_{i \neq j}^{4} \frac{r_{j}}{r_{i} r_{i j}} \frac{\sin \left(\theta_{j}\right)}{\sin \left(\theta_{i}\right)} \sin \left(\varphi_{i}-\varphi_{j}\right) \frac{\partial^{2}}{\partial \varphi_{i} \partial r_{i j}} .
\end{aligned}
$$




\section{Method of calculations and trial wave functions}

Our calculations are based on using the VMC method which is regarded as one of the most important tools in studying atoms and molecules. It is built on a combination of two ideas. Namely, (i) the variational principle and the Monte Carlo evaluation of integrals using importance sampling based on the Metropolis algorithm and (ii) the VMC method is utilized to compute the quantum expectation values of an operator with a given trial wave function. Given a Hamiltonian operator and a trial wave function, it is then possible to calculate the least energy eigenvalues of a given state of an atom with respect to the parameters of the used trial wave function. This approach is explained briefly in the previous works of Doma et al. [10, 12, 14-16].

The ground-state trial wave function for the $\mathrm{Be}$ atom symbolically written has the form of

$$
\psi(\mathrm{Be})=\psi_{0} f\left(r_{12} r_{13} r_{14} r_{23} r_{24} r_{34}\right),
$$

where $\psi_{0}$ is the SLD function and $f$ is the correlation function due to the interactions between the electrons. If we neglect the interactions between the electrons, then the ground-state trial wave function with the total spin $S=0$ and its $z$-component $S_{z}=0$ may be symbolically expressed by

$$
\begin{aligned}
& \psi_{0}=\frac{1}{\sqrt{4 !}}
\end{aligned}
$$

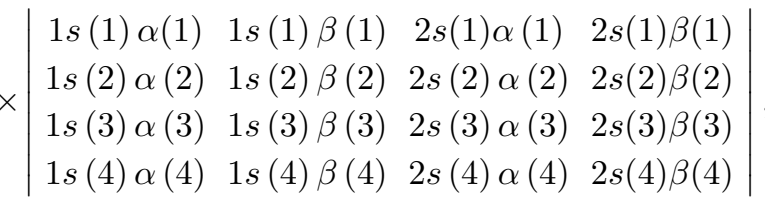

where $\alpha(i)$ and $\beta(i)$ represent the spin-up and spindown functions of the $i$-th electron, respectively. The functions $1 s(i)$ and $2 s(i)$, in turn, stand for the orbital functions of the states $(n, l)=(1,0)$ and $(2,0)$ of the $i$-th electron, respectively. In the calculations, we used two types of orbital functions. The first is the hydrogenic function and the second is a modified hydrogenic function. It is well known that the hydrogenic function constitutes a sufficient basis for energy calculation. Trial wave functions constructed from hydrogenic basis are used to study the beryllium atom under the compression effect neglecting electron-electron correlation [8, 19] with good results. Accordingly, we use these functions in constructing the first two trial wave functions in our calculations. These functions are given, with the usual notations, in a.u., by

$$
1 s=\frac{1}{\sqrt{\pi}} Z^{3 / 2} \mathrm{e}^{-Z r}
$$

and

$$
2 s=\frac{1}{\sqrt{\pi}}\left(\frac{Z}{2}\right)^{3 / 2}\left(1-\frac{Z r}{2}\right) \mathrm{e}^{-\frac{Z r}{2}} .
$$

We will treat $Z$ as a variational parameter and denote it by $Z^{\prime}$.
By some simple algebra and substituting for the hydrogen wave functions $1 s(i)$ and $2 s(i)$, we can easily obtain the function $\psi_{0}$. As a result, $\psi_{0}$ is a linear combination of terms constructed from the single-particle hydrogenic wave functions in such a way that the total spin of the system is $S=0$ and its $z$-component is $M_{S}=0$.

For the correlation function $f$, we used two different types of correlations. The first one is the Jastrow correlation function which is a product of exponential functions [20], i.e.,

$$
f_{1}=\prod_{1=i<j}^{4} \exp \left(\frac{r_{i j}}{l\left(1+k r_{i j}\right)}\right),
$$

where $k$ is the variational parameter. To satisfy the cusp conditions [21], we assume that

$$
l= \begin{cases}4 & \text { for like spins } \\ 2 & \text { for unlike spins }\end{cases}
$$

hence $k_{1}$ is for the like spins and $k_{2}$ for the unlike spins. Therefore, our first trial wave function is given by

$$
\psi_{1}=\psi_{0} f_{1}
$$

and it contains three variational parameters, i.e., $k_{1}, k_{2}$ and $Z^{\prime}$.

The second correlation function is a product of the following functions [22]:

$$
f_{2}=\prod_{1=i<j}^{4}\left(1+A r_{i j} \exp \left(-B r_{i j}\right)\right) \text {. }
$$

where $B$ is the variational parameter. To satisfy the cusp conditions we assume that

$$
A=\left\{\begin{array}{l}
\frac{1}{4} \text { for the like spins, } \\
\frac{1}{2} \text { for the unlike spins. }
\end{array}\right.
$$

hence $B_{1}$ is for the like spins and $B_{2}$ for the unlike spins. Therefore, our second trial wave function is given by

$$
\psi_{2}=\psi_{0} f_{2}
$$

and it contains three variational parameters, i.e., $B_{1}, B_{2}$ and $Z^{\prime}$.

Furthermore, we constructed the third trial wave function $\psi_{3}$ using the modified radial hydrogenic wave functions [23] in the SLD (4) and where the correlation function is $f_{2}$ while the function $1 s$ is given by (5). Then,

$$
2 s=\frac{1}{\sqrt{4 \pi}} \frac{\sqrt{Z}^{\prime 3}}{\sqrt{8 c_{0}^{2}-12 c_{0}+6}}\left(2 c_{0}-\frac{Z^{\prime} r}{2}\right) \mathrm{e}^{-\frac{z^{\prime} r}{2}},
$$

where $Z^{\prime}$ and $c_{0}$ are variational parameters. Hence, $\psi_{3}$ contains four variational parameters, i.e., $c_{0}, B_{1}$, $B_{2}$ and $Z^{\prime}$.

\section{Results and discussion}

Our investigations in the present paper are based on applying the VMC method to find the least energy eigenvalue of the ground-state of the beryllium atom. To achieve this goal, we used three trial wave 


\section{TABLE I}

Values of the parameters which produced the best values of the ground- state energy eigenvalue of beryllium.

\begin{tabular}{c|c|c|c}
\hline \hline & $\psi_{1}$ & $\psi_{2}$ & $\psi_{3}$ \\
\hline$Z^{\prime}$ & 3.965 & 3.2885 & 3.2885 \\
$k_{1}$ & 2.2 & - & - \\
$k_{2}$ & 0.135 & - & - \\
$B_{1}$ & - & 0.001 & 0.001 \\
$B_{2}$ & - & 0.88 & 0.88 \\
$c_{0}$ & - & - & 2.7
\end{tabular}

TABLE II

Ground-state energy eigenvalue of beryllium $E$ by using the three trial wave functions. The values of the standard deviations are given.

\begin{tabular}{c|c|c}
\hline \hline & $E$ [a.u.] & $\sigma$ \\
\hline$\psi_{1}$ & -14.6557 & 0.0013 \\
$\psi_{2}$ & -14.664401 & 0.0013 \\
$\psi_{3}$ & -14.667313 & 0.0019 \\
exact [24] & -14.667356508 & - \\
previous [25] & -14.66735 & -
\end{tabular}

functions which are in the form of SLD functions multiplied by correlation functions. Two types of correlation functions are considered. In these calculations, the SLD with modified hydrogenic radial functions multiplied by our second correlation function is also used. All energies are given in atomic units with a set of $2.5 \times 10^{7}$ Monte Carlo points to make the statistical error as low as possible. Since the principle of the VMC method depends on finding the minimum energy by using the appropriate trial wave function, therefore in this study we focus on finding a simple trial wave function which can produce a minimum energy eigenvalue for the ground-state of beryllium in good agreement with the corresponding exact value and accompanied by the least possible standard deviation.

In Table I, we present the parameter values of the three trial wave functions which produced the best values of the calculated ground-state energy and standard deviations of the Be atom.

The corresponding values of the ground-state energy eigenvalues of beryllium calculated for the best three trial wave functions are given in Table II. The corresponding values of standard deviations are also given. The exact value and the previous value are also included in Table II.

In Fig. 1, we present the variation of the groundstate energy of the beryllium atom with respect to two parameters $Z^{\prime}$ and $k_{1}$. For this case, the first trial wave function $\psi_{1}$ is used.

The variations of the standard deviation associated with the ground-state energy of beryllium using $\psi_{1}, \psi_{2}$ and $\psi_{3}$, with respect to the number of VMC points, are provided in Fig. 2.

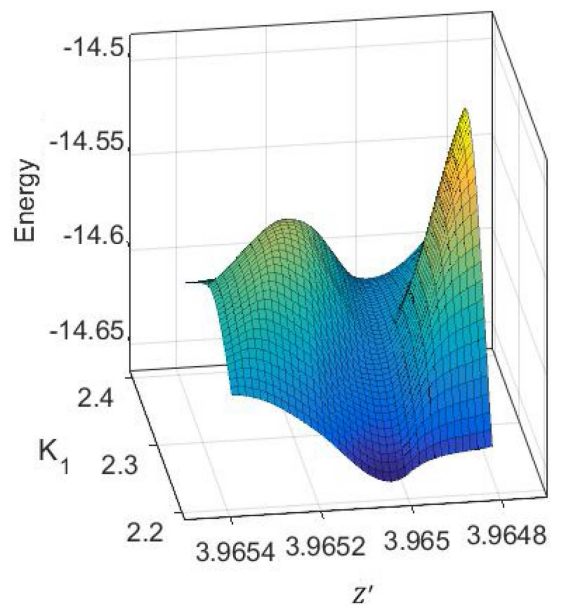

Fig. 1. Variations of the ground-state energy of beryllium, calculated by using the trial wave function $\psi_{1}$ with respect to the parameters $Z^{\prime}$ and $k_{1}$. The value of $k_{2}$ is 0.135 .

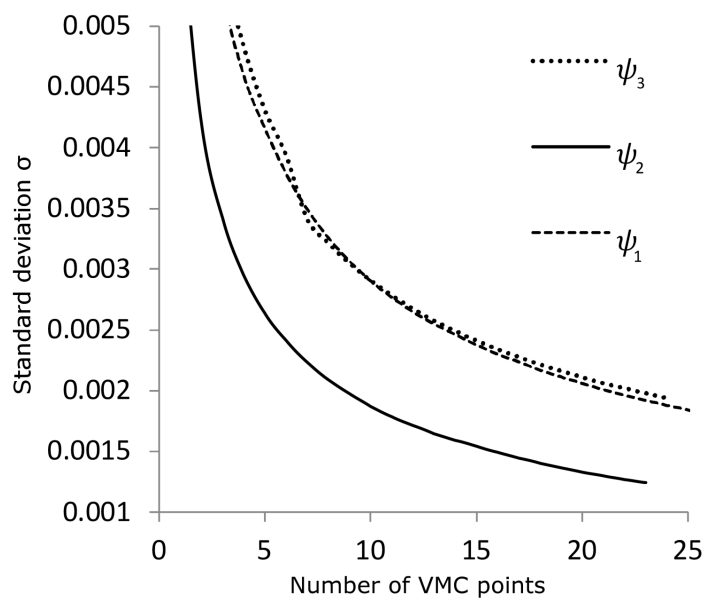

Fig. 2. Variations of the standard deviation associated with the ground-state energy of beryllium with respect to the number of VMC points by using $\psi_{1}, \psi_{2}$ and $\psi_{3}$.

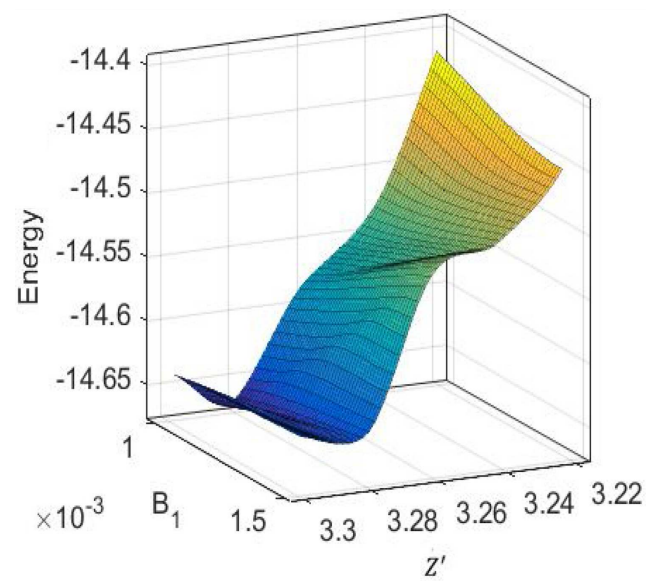

Fig. 3. Variations of the ground-state energy of beryllium, calculated by using the trial wave function $\psi_{2}$ with respect to the parameters $Z^{\prime}$ and $B_{1}$. The value of $B_{2}$ is 0.88 . 
TABLE III

Mean distance of the electrons from the nucleus and the mean distance between the electrons in the ground-state of beryllium.

\begin{tabular}{c|c|c}
\hline \hline & $\left\langle r_{i}\right\rangle$ & $\left\langle r_{i j}\right\rangle$ \\
\hline$\psi_{1}$ & 1.079 & 1.7463 \\
$\psi_{2}$ & 1.41286 & 2.31126 \\
$\psi_{3}$ & 1.10045 & 1.7820 \\
previous [24] & 1.49319434912 & 2.5454427433
\end{tabular}

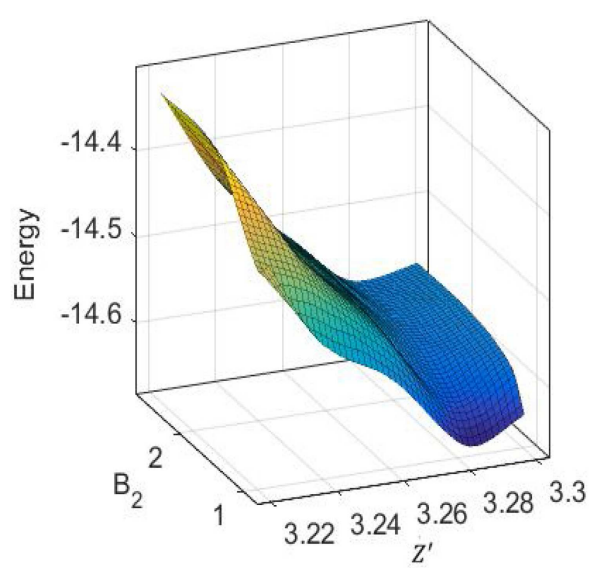

Fig. 4. Variations of the ground-state energy of beryllium, calculated by using the trial wave function $\psi_{2}$ with respect to the parameters $Z^{\prime}$ and $B_{2}$. The value of $B_{1}$ is 0.001 .

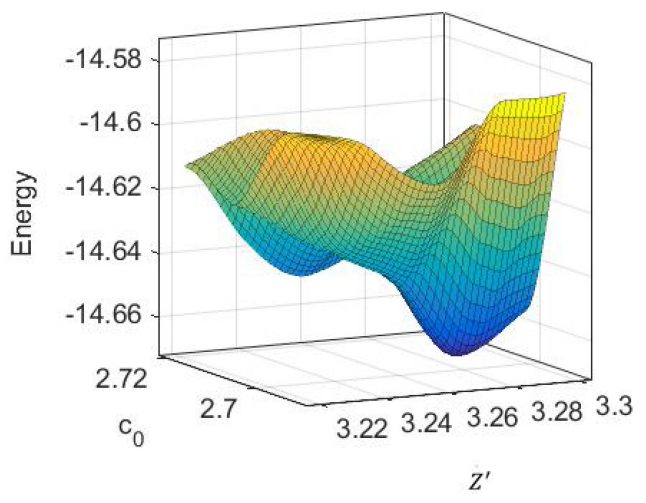

Fig. 5. Variations of the ground-state energy of beryllium, calculated by using the trial wave function $\psi_{3}$ with respect to the parameters $Z^{\prime}$ and $c_{0}$. $B_{1}=0.001$ and $B_{2}=0.88$.

The behavior of the ground-state energy of the Be atom as a function of $Z^{\prime}$ with respect to the parameter $B_{1}$ when the value of $B_{2}$ is given, and with respect to $B_{2}$ when $B_{1}$ is given, is presented in Figs. 3 and 4 , respectively. These results were obtained with the use of the $\psi_{2}$ trial function.

Figure 5 demonstrates the change of the groundstate energy of the beryllium atom calculated using the trial wave function $\psi_{3}$. The energy dependence is shown for the parameters $Z^{\prime}$ and $c_{0}$.
To check the accuracy of the three used trial wave functions in achieving good results for other groundstate characteristics of the beryllium atom, we calculated the mean distance of the electrons from the nucleus and the mean distance between the electrons. These are given according to

$$
\left\langle r_{i}\right\rangle=\frac{1}{n} \frac{\left\langle\psi\left|\sum_{i=1}^{n} r_{i}\right| \psi\right\rangle}{\langle\psi \mid \psi\rangle}
$$

and

$$
\left\langle r_{i j}\right\rangle=\frac{2}{n(n-1)} \frac{\left\langle\psi\left|\sum_{i<j}^{n} r_{i j}\right| \psi\right\rangle}{\langle\psi \mid \psi\rangle},
$$

where $n=4$.

In Table III, we present the mean distance of the electrons from the nucleus $\left\langle r_{i}\right\rangle$ and the mean distance between the electrons $\left\langle r_{i j}\right\rangle$. Corresponding previous values reported in [24] are also given in Table III.

\section{Conclusion}

In this paper, we considered the VMC method which has been proven to be a very powerful tool for studying quantum systems in the physics field, atomic and molecular physics in particular. The VMC technique provides a simple, robust and efficient way to solve the ground-state energy of a quantum many-particle system. Since the method is relatively insensitive to the size of the system, it can be applied to large systems; where some other methods are computationally not feasible. The major advantage of the VMC method is the possibility to freely choose the analytical form of the trial wave function, which may contain a highly sophisticated term, in such a way that the electron correlation is explicitly considered. This is an important feature valid for the VMC methods which are therefore extremely useful to study physical cases, where the electron correlation plays a crucial role. For these reasons, this method can be extended to study much more complex many-body quantum mechanical systems involving higher dimensions.

Accordingly, we studied the effect of three different types of correlation functions associated with trial wave functions of the form of the SLD for the configuration of the ground state of the beryllium atom. Furthermore, to check the suitability of using these trial wave functions in the calculations of other characteristics of this atom, with the least number of variational parameters, we used our three optimized trial wave functions in the calculations of the mean distance of the electrons from the nucleus and the mean distance between the electrons in the ground-state of beryllium. In conclusion, we noticed that the obtained result concerning the ground-state energy eigenvalue of the beryllium atom by using the third trial wave function $\psi_{3}$ is in better agreement with the corresponding exact value rather than the other two trial wave functions, 
while the standard deviations by using the functions $\psi_{1}$ and $\psi_{2}$ are better than the corresponding one by using $\psi_{3}$.

The obtained results also showed that the VMC method can be applied successfully for the investigation of the ground-state characteristics of the beryllium atom by a suitably chosen trial wave function, which takes into account the correlation part, due to the interactions between the electrons and the cusp condition.

\section{References}

[1] H. de Oliveira Batael, E.D. Filho, Theor. Chem. Acc. 137, 65 (2018).

[2] D.A. Glazov, A.V. Malyshev, A.V. Volotka, V.M. Shabaev, I.I. Tupitsyn, G. Plunien, Nucl. Instrum. Methods Phys. Res. $B$ 408, 46 (2017).

[3] A.W. King, A.L. Baskerville, H. Cox, Philos. Trans. R. Soc. A 376, 1 (2018).

[4] R. Gobato, A. Heidari, A. Mitra, M.R.R. Gobato, Am. J. Quant. Chem. Mol. Spectrosc. 2, 9 (2018).

[5] L.G. Jiao, L.R. Zan, L. Zhu, Y.K. Ho, Computat. Theor. Chem. 1135, 1 (2018).

[6] N.S. Blunt, E. Neuscamman, J. Chem. Theory Comput. 15, 178 (2018).

[7] S.L. Davis, J. Chem. Educat. 84, 711 (2007).

[8] A.D. Sa nu-Ginarte, L. Ferrer-Galindo, R.A. Rosas, A. Corella-Madueño, Ri. Betancourt-Riera, L.A. Ferrer-Moreno, R. Riera, J. Phys. Commun. 2, 015001 (2018).

[9] F. Wu, L. Meng, Chin. Phys. B 27, 9 (2018).

[10] S.B. Doma, M.O. Shaker, A.M. Farag, F.N. El-Gammal, Indian J. Phys. 92, 279 (2018).
[11] C.J. Umrigar, M.P. Nightingale, K.J. Runge, J. Chem. Phys. 99, 2865 (1993).

[12] S.B. Doma, M.O. Shaker, A.M. Farag, F.N. El-Gammal, Acta Phys. Pol. A 126, 700 (2014).

[13] S.A. Alexander, R.L. Coldwell, Int. J. Quantum Chem. 63, 1001 (1997).

[14] S.B. Doma, M.O. Shaker, A.M. Farag, F.N. El-Gammal, J. Exp. Theor. Phys. 124, 1 (2017).

[15] S.B. Doma, F.N. El-Gammal, A.A. Amer, Mol. Phys. 116, 1827 (2018).

[16] S.B. Doma, M. Abu-Shady, F.N. El-Gammal, A.A. Amer, Mol. Phys. 114, 1787 (2016).

[17] A.M. Frolov, D.M. Wardlaw, Phys. Rev. A 78, 042506 (2008).

[18] M.B. Ruiz, Int. J. Quant. Chem. 101, 246 (2005).

[19] A.D. Sa nu-Ginarte, E.M. Guillén-Romero, L. Ferrer-Galindo, L.A. Ferrer-Moreno, Ri. Betancourt-Riera, R. Riera, Results Phys. 13, 102261 (2019).

[20] C. Filippi, C.J. Umrigar, J. Chem. Phys. 105, 213 (1996).

[21] T. Kato, Commun. Pure Appl Math. X, 151 (1957).

[22] J.O. Hirschfelder, J. Chem. Phys. 39, 3145 (1963).

[23] H.S. Zhong, M. Kun, Y.J. Ming, L. Fen, Chin. Phys. 17, 11 (2008).

[24] I. Hornyák, L. Adamowicz, S. Bubin, Phys. Rev. A 100, 032504 (2019).

[25] M. Przybytek, M. Lesiuk, Phys. Rev. A 98, 062507 (2018). 